\section{Psicologia Escolar \\ e Educacional}

ARTIGO

DOI: http://dx.doi.org/10.1590/2175-35392020217008

Elocid - e217008

\title{
DESENVOLVIMENTO DE HABILIDADES SOCIAIS EM EAD: O PAPEL DO FEEDBACK DO TUTOR
}

\author{
Joene Vieira-Santos ${ }^{1} \mathbb{D}$
}

\section{RESUMO}

A efetividade do treinamento de habilidades sociais (THS) presencial está bem estabelecida na literatura, mas são escassos estudos que avaliam o resultado de cursos na modalidade de Educação à Distância (EaD) destinados a promover essas habilidades. Nesses cursos, o tutor assume um papel importante ao orientar o desempenho dos alunos. Portanto, este estudo avaliou como o feedback fornecido pelo tutor afeta as notas do aluno em um THS em EaD. Os feedbacks foram classificados quanto a presença, amplitude, efeito, função e organização. Os dados parecem indicar que nem a presença nem o tipo de feedback afetam o desempenho dos alunos. Contudo, os dados também sinalizaram que alguns feedbacks foram apagados e/ou alterados após a atividade ser refeita. Novos estudos, com amostras maiores e analisando o histórico do feedback fornecido pelo tutor a cada tarefa, poderão avaliar com maior precisão como o feedback interfere no desenvolvimento das habilidades sociais dos participantes.

Palavras-chave: Feedback; treinamento de habilidades sociais; educação à distância.

\section{Development of social skills in DE: the role of tutor's feedback}

\begin{abstract}
The effectiveness of face-to-face social skills training (SST) is well established in the literature, but there are few studies that evaluate the results of distance education courses (DE) aimed at promoting these skills. In these courses, the tutor assumes an important role in guiding student performance. Therefore, this study evaluated how the feedback provided by the tutor affects the student's grades in a SST in the DE. The feedbacks were classified according to presence, amplitude, effect, function and organization. The data seems to indicate that neither the presence nor the type of feedback affects students' performance. However, the data also signaled that some feedbacks were deleted and / or changed after the activity was redone. New studies, with larger samples and analyzing the history of the feedback provided by the tutor for each task, will be able to evaluate with greater precision how the feedback interferes in the development of the social skills of the participants.
\end{abstract}

Keywords: Feedback; social skills training; distance education.

\section{Desarrollo de habilidades sociales en EaD: el papel del feedback del tutor}

\section{RESUMEN}

La efectividad del entrenamiento de habilidades sociales (EHS) presencial está bien establecida en la literatura, pero son escasos estudios que avalúen el resultado de cursos en la modalidad de Educación a Distancia (EaD) destinados a promover esas habilidades. En esos cursos, el tutor asume un papel importante al orientar el rendimiento de los alumnos. Por lo tanto, este estudio evaluó cómo el fornecido por el tutor afecta las notas del alumno en un EHS en EaD. Los feedbacks fueron clasificados cuanto a presencia, amplitud, efecto, función y organización. os datos parecen indicar que ni la presencia ni el tipo de feedback afectan el rendimiento de los alumnos. Sin embargo, los datos también señalaron que algunos feedbacks fueron borrados y/o alterados tras la actividad hacerse otra vez. Nuevos estudios, con más muestreos y analizando el histórico del feedback suministrado por el tutor a cada tarea, podrán evaluar con más precisión como el feedback interfiere en el desarrollo de las habilidades sociales de los participantes.

Palabras clave: Feedback; entrenamiento de habilidades sociales; educación a distancia.

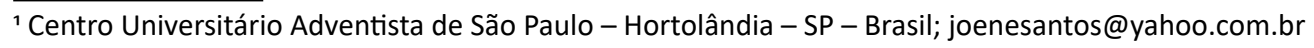




\section{INTRODUÇÃO}

Nas últimas décadas tem se destacado a importância de proporcionar às crianças, dentro do ambiente escolar, condições para desenvolver as habilidades socioemocionais (Abed, 2016). Tais habilidades são compreendidas como um construto multidimensional relacionado a "adquirir e aplicar efetivamente os conhecimentos, atitudes e habilidades necessárias para entender e gerenciar emoções, definir e alcançar objetivos positivos, sentir e mostrar empatia pelos outros, estabelecer e manter relacionamentos positivos e tomar decisões responsáveis" (Domitrovich, Durlak, Staley, \& Weissberg, 2017, p. 408, tradução livre).

Nesse contexto, o professor desempenha um papel relevante, pois cabe a ele promover um ambiente no qual seus alunos possam desenvolver tanto as habilidades cognitivas como as habilidades socioemocionais (Domitrovich et al., 2017; Rosin-Pinola, Marturano, Elias, \& Del Prette, 2017; Weissberg, Durlak, Domitrovich, \& Gullotta, 2015). No entanto, para que isso ocorra, é necessário que, entre outros fatores, o professor apresente um repertório elaborado de habilidades sociais (Del Prette, Del Prette, Torres, \& Pontes, 1998; Del Prette \& Del Prette, 1997; Rosin-Pinola et al., 2017). Segundo Z. A. P. Del Prette e Del Prette (2010, p. 31), "o termo habilidades sociais refere-se à existência de diferentes classes de comportamentos sociais no repertório do indivíduo para lidar de maneira adequada com as demandas das situações interpessoais". Esses comportamentos caracterizam-se por (a) serem valorizados por uma determinada cultura, (b) possuírem alta probabilidade de produzir resultados positivos para todos os envolvidos (indivíduo, interlocutor e comunidade) e (c) contribuírem para um desempenho socialmente competente (Del Prette \& Del Prette, 2017a).

Segundo Del Prette (2016), já há algum tempo se tem defendido a existência de processos de assessoria direcionados para o desenvolvimento das habilidades sociais dos professores de tal forma que esses profissionais estejam melhor capacitados tanto para promover uma formação mais ampla dos alunos (o que abrangeria as habilidades socioemocionais), como para a promoção da inclusão no ambiente escolar. Os programas de treinamento de habilidades sociais destinados à formação continuada dos professores são uma forma realizar essa assessoria (Corrêa, 2008; A. Del Prette et al., 1998; Z. A. P. Del Prette et al., 1998; Del Prette \& Del Prette, 1997; Vila, 2005).

De acordo com A. Del Prette e Del Prette (2010, p. 128), o programa de treinamento de habilidades sociais (THS) é compreendido como

...um conjunto de atividades planejadas que estruturam processos de aprendizagem, mediados e conduzidos por um terapeuta ou coordenador, visando: (a) ampliar a frequência e/ou melhorar a proficiência de habilidades sociais já aprendidas, mas deficitárias, (b) ensinar habilidades sociais novas significativas, (c) diminuir ou extinguir comportamentos concorrentes com tais habilidades.

O THS, conforme sugerem A. Del Prette e Del Prette (2010), apoia-se em três princípios fundamentais: (a) as habilidades sociais são aprendidas e, portanto, podem ser ensinadas; (b) a aprendizagem dessas habilidades pode ocorrer de forma acidental ou pode ser planejada por meio de condições estruturadas; e (c) as habilidades sociais possuem um caráter situacional-cultural e, portanto, devem ser pensadas a partir das práticas que são valorizadas em determinados contextos.

Outro aspecto importante do THS refere-se ao modelo adotado. Segundo Del Prette e Del Prette (2011), existem dois modelos principais: o modelo terapêutico e o modelo pedagógico. O modelo terapêutico refere-se à aplicação de THS à população de pacientes com transtornos psiquiátricos e caracteriza-se, principalmente, pelo uso dos procedimentos de instrução, modelação e modelagem. Já o modelo pedagógico, conforme apontam esses autores, refere-se à aplicação de THS às populações não-clínicas (ou seja, sem diagnóstico de transtornos psiquiátricos), voltados para a promoção de habilidades sociais como meio de melhorar a qualidade das relações interpessoais e, consequentemente, a qualidade de vida dos participantes. Os autores destacam que esse modelo é caracterizado pelo esquema de aprendizagem olhar-avaliar-copiar, de tal forma que os participantes possam aprender a agir de maneira semelhante aos modelos apresentados.

Independente do modelo, o THS compreende duas etapas: avaliação e intervenção (Murta, 2005; Del Prette \& Del Prette, 1999). A avaliação busca levantar os recursos e déficits de habilidades sociais e as variáveis situacionais (antecedentes, consequências, contexto, interlocutores e normas culturais), pessoais (cognitivas, motivacionais, papéis sociais, etc.) e sociodemográficas (idade, gênero, escolaridade, etc.) do participante por meio de métodos diretos (observação, auto-observação e autorregistro fisiológico) e indiretos (avaliação por relatos de outros ou autorrelatos) (Del Prette \& Del Prette, 2009). Já a intervenção consiste nos procedimentos e técnicas empregados ao longo das sessões do THS, sendo que os mais utilizados são "fornecimento de instruções, ensaio comportamental, modelação, modelagem, feedback verbal e em vídeo, tarefas de casa, reestruturação cognitiva, solução de problemas, relaxamento ...e, em caso de intervenções grupais, vivências" (Murta, 2005, p. 284). As vivências são atividades estruturadas, análogas às situações presentes no cotidiano, que permitem ao facilitador observar desempenhos específicos dos participantes e estabelecer condições 
para a aquisição e/ou fortalecimento de habilidades sociais (A. Del Prette \& Prette, 2010).

Também é importante destacar que no THS o facilitador ou coordenador possui um papel importante no processo de condução do programa. Ele precisa dispor de um repertório elaborado de habilidades sociais que o permita: observar, demonstrar empatia e disposição para ajudar, apresentar feedback, encorajar os participantes, coordenar o grupo, fornecer informações, entre outras (Z. A. P. Del Prette \& Del Prette, 2010).

Os estudos que descrevem a aplicação de THS junto a professores da Educação Básica têm demonstrado que esse tipo de programa, ao proporcionar condições para que o docente desenvolva habilidades sociais, resulta em mais diálogo e oportunidades de participação ativa dos alunos em sala (Z. A. P. Del Prette et al., 1998; Del Prette \& Del Prette, 1997), melhoras na relação professor-aluno e na disciplina em sala (Corrêa, 2008) e mais expressões de afeto do professor para com o aluno (A. Del Prette et al., 1998; Vila, 2005). Esses treinamentos têm sido realizados dentro do modelo pedagógico, em grupo, na modalidade presencial e com o uso de vivências.

\section{A oferta de THS na modalidade híbrida e suas implicações}

Os resultados do THS, descritos na literatura, parecem ser bastante desejáveis no contexto escolar. Assim sendo, na tentativa de ampliar o acesso dos professores a esse tipo de intervenção, o Grupo Relações Interpessoais e Habilidades Sociais da Universidade Federal de São Carlos (RIHS/UFSCar), em parceria com Secretaria de Educação a Distância da UFSCar (SEaD/UFSCar), elaborou o Programa de Habilidades Sociais na Escola Mediadas pelo Professor. O programa foi proposto para ser ofertado na modalidade híbrida (Vaughan, Cleveland-Innes, \& Garrison, 2013), envolvendo atividades no ambiente virtual de aprendizagem (AVA) e encontros presenciais semanais para apresentar e discutir os conteúdos de cada unidade.

Em relação à sua estrutura, o programa foi constituído de três módulos teórico-práticos - ou seja, que envolvem atividades teóricas (cognitivas) e atividades práticas (tarefas interpessoais) - e um módulo de prática supervisionada na condução atividades em sala de aula (Del Prette, 2016). Os módulos foram denominados da seguinte forma: Módulo I - Programa de Habilidades Sociais (PHS), Módulo II - Programa de Habilidades Sociais na Infância (PHSI), Módulo III - Programa de Habilidades Sociais Educativas (PHSE) e Módulo IV Programa de Desenvolvimento Socioemocional dos alunos na escola (PDSE).

A oferta de um THS na modalidade híbrida, voltado para a formação continuada de professores, pode trazer algumas implicações que, a princípio, parecem não existir nos programas presenciais com professores (descritos acima). A primeira implicação refere-se ao fato de o programa ser planejado no formato de um curso, dando maior ênfase ao papel instrucional do THS. Isso conduz a três desdobramentos importantes. O primeiro refere-se à presença de mais conteúdo acadêmico (textos e tarefas teóricas de caráter cognitivo) e menos atividades práticas quando comparado aos THS realizados na modalidade presencial. $O$ segundo diz respeito à necessidade de que seja atribuída uma nota às atividades realizadas pelo participante, de tal forma que seja possível avaliar a aprovação (ou não) do mesmo no curso. $O$ terceiro desdobramento é o desafio para adaptar o modelo de aprendizagem olhar-avaliar-copiar e o uso de vivências para o AVA. Isso porque é necessário pensar em atividades que permitam ao participante praticar suas habilidades sociais a partir do contato com modelos que sinalizam desempenhos socialmente competentes diante de diferentes demandas interpessoais do contexto escolar.

A segunda implicação diz respeito ao papel de coordenador ou facilitador. Enquanto no THS presencial existem um ou dois facilitadores que se revezam nas funções de conduzir as atividades e observar o desempenho dos participantes nas atividades propostas (Del Prette \& Del Prette, 1999), em um curso híbrido existe a presença de múltiplos facilitadores. Assim como outros cursos realizados na modalidade híbrida, o programa (descrito no presente estudo) contou com uma equipe polidocente para o seu funcionamento. Segundo Mill (2017), o termo polidocência refere-se a uma categoria explicativa empregada para examinar a condição docente na Educação à Distância ( $\mathrm{EaD})$, a qual é, geralmente, caracterizada por uma atuação colaborativa e fragmentada de um grupo de distintos profissionais. De acordo com esse autor, a quantidade de membros na equipe e as funções desempenhadas por esses membros podem variar; contudo, o grupo de profissionais envolvidos na polidocência é composto por “... docente-autor (ou docente-conteudista), docente-formador (ou docente-aplicador), docente-tutor virtual (ou docente-tutor a distância), docente-tutor presencial, projetista educacional (ou designer instrucional), equipe multidisciplinar, equipe coordenadora etc." (Mill, 2017, p. 11).

Pensando especificamente no curso em questão, a condução do THS propriamente dita contou com dois grupos de docentes: (a) docentes-formadores, responsáveis por conduzir os encontros presenciais, e (b) docentes-tutores, responsáveis por acompanhar os alunos no AVA, fornecendo feedback para as tarefas realizadas. Nesse sentido, um desdobramento importante relacionado à presença de diferentes facilitadores refere-se à avaliação realizada do desempenho do cursista. Apesar de haver critérios estabelecidos sobre o desempenho esperado para cada tarefa, a avaliação 
do desempenho do aluno na tarefa, a atribuição de notas e aquilo que é enfatizado no feedback fornecido pelo tutor, inevitavelmente, envolve um componente subjetivo: a percepção do tutor. Portanto, a existência de múltiplos tutores pode implicar na existência de níveis de exigências distintos para o desempenho do aluno, o que pode resultar em diferenças nas avaliações e no desenvolvimento das habilidades sociais.

O tutor desempenha um papel importante nos cursos na modalidade híbrida ou de EaD, visto que assume "a função de apoiar o estudante ao longo do seu processo de formação, de maneira personalizada" (Silva, 2017, p. 8). Nessa direção, uma tarefa importante do tutor é o fornecimento de feedback ao desempenho dos alunos. Existem múltiplas definições de feedback (Abreu-e-Lima \& Alves, 2011; Archer, Crispim, \& Cruz, 2016; Z. A. P. Del Prette \& Del Prette, 2010; Shute, 2008), mas o ponto central em todas elas refere-se ao fato de que o feedback consiste em apresentar informações sobre o desempenho de uma pessoa, ou seja, sobre comportamentos específicos apresentados pelo indivíduo.

O feedback pode ser classificado de várias formas. Por exemplo, Del Prette e Del Prette (2017b) sugerem que o feedback pode ser classificado em função do efeito que produz sobre o comportamento do outro, podendo ser considerado como positivo (quando valoriza o comportamento, contribuindo para a manutenção ou aumento de sua frequência) ou negativo (quando reprova o comportamento, podendo contribuir para a redução de sua frequência). Uma forma especial de combinar esses dois efeitos é o que tem sido denominado de feedback sanduíche, o qual consiste na apresentação da seguinte sequência: (a) um aspecto positivo do desempenho do indivíduo, (b) um aspecto que precisa ser melhorado (aspecto negativo) e (c) um outro aspecto positivo (Abreu-e-Lima \& Alves, 2011; Z. A. P. Del Prette \& Del Prette, 2010).

A partir das contribuições de Shute (2008), o feedback pode também ser classificado quanto a amplitude, a função e a forma de organizá-lo. Quanto à amplitude, o feedback pode ser geral (comentários gerais sobre o desempenho do aluno) ou específico (enfatizando aspectos específicos do desempenho do aluno). Quanto à função, o feedback pode ser de verificação (um julgamento simples a respeito da resposta estar correta ou não) ou de elaboração (um conjunto de dicas e orientações sobre como chegar ao resultado esperado). Quanto à forma de organizar, o feedback pode ser diretivo (apontando o que precisa ser corrigido ou revisado) ou facilitador (apresentando comentários e sugestões para orientar o aluno no processo de revisão de sua atividade).

Apesar das diferentes formas de classificar um feedback, ao escrevê-lo, o tutor precisar ter, como preocupação principal, fornecer informações para o aluno que contribuam para a melhoria do seu desempenho.
Nessa direção, Shute (2008) sugere que, ao escrever o feedback, o tutor deve levar em consideração que (a) o foco deve estar sobre a tarefa realizada, (b) deve ser claro e específico, (c) diminuir as dúvidas entre o desempenho do aluno e os objetivos da tarefa e (d) encorajar o aluno a prosseguir no processo de aprendizagem, (e) não deve ser longo e com muitas informações e (f) nem conter comparações entre estudantes.

\section{O presente estudo}

A efetividade de programas de THS realizados presencialmente já está bem estabelecida na literatura (Z. A. P. Del Prette \& Del Prette, 2011), mas ainda são escassos estudos que avaliem o resultado de cursos híbridos destinados a promover o desenvolvimento de tais habilidades. Aliado a isso, sabe-se que o feedback fornecido pelo facilitador ou coordenador do THS, na modalidade presencial, é extremamente importante para a aquisição e/ou aperfeiçoamento das habilidades abordadas (Z. A. P. Del Prette \& Del Prette, 2010), mas ainda não existem dados que apontem se o feedback do tutor desempenharia a mesma função. Portanto, em uma tentativa inicial de examinar esse fenômeno, o presente estudo tem como objetivo avaliar como o feedback fornecido pelo tutor afeta as notas do aluno ao longo das atividades propostas em um curso, na modalidade híbrida, destinado à promoção do desenvolvimento de habilidades sociais em professores da Educação Básica. Além disso, são objetivos secundários:

1. Identificar as subclasses de feedback predominantes, tomando como base a classificação destes quanto à presença (ou ausência), amplitude, efeito, função e organização;

2. Examinar se existem diferenças nas notas das tarefas em função da presença do feedback e das diferentes possibilidades de classificá-los;

3. Verificar se existem diferenças nas notas em função do tipo de tarefa (tarefas acadêmicas e tarefas de interação interpessoais);

4. Avaliar se existem diferenças na frequência das subclasses de cada classificação do feedback (amplitude, efeito, função e organização) em função do tipo de tarefa.

\section{MÉTODO}

Neste estudo foram analisados os dados do Módulo I do Programa Habilidades sociais na escola mediada pelo professor, o qual ocorreu no período de 06/05/2016 a 05/07/2016. O projeto foi aprovado pelo Comitê de Ética em Pesquisa com Seres Humanos da Universidade Federal de São Carlos (protocolo CAAE 50409115.4.0000.5504) e os participantes, por meio da assinatura do Termo de Consentimento Livre-Esclarecido, autorizaram que as informações fornecidas fossem utilizadas para fins de pesquisa, desde que fosse 
preservado o anonimato dos mesmos. Além disso, a coordenadora responsável pelo projeto também forneceu autorização para que a pesquisadora acessasse o sistema e extraísse as informações necessárias para a presente pesquisa.

\section{Participantes}

Fizeram parte deste trabalho dois grupos de participantes: os cursistas e seus respectivos tutores. Os cursistas foram 37 professores da rede pública de Educação Fundamental de uma cidade do interior de São Paulo, que foram convidados a participar do projeto através da Secretaria Municipal de Educação.

Os tutores foram 14 psicólogos com experiência em docência presencial e em pesquisas no campo teórico-prático das habilidades sociais. Cada cursista foi acompanhado por um único tutor. E os tutores receberam um manual de orientação sobre como agir no AVA.

\section{Materiais}

O Módulo I foi composto por seis unidades de ensino. Cada unidade era composta por um texto didático (disponível no AVA), atividades no AVA e uma aula presencial (que ocorria uma vez por semana). As atividades eram de dois tipos: (a) tarefas acadêmicas de casa (TAC), que correspondiam a atividades conceituais, e (b) tarefas interpessoais de casa (TIC), que correspondiam a atividades de interação com outras pessoas nas quais o cursista tinha que colocar em prática uma das habilidades abordadas na unidade. Os dados gerados a partir das tarefas e analisados no presente estudo foram: (a) as notas do aluno e (b) os feedbacks dos tutores sobre o desempenho dos cursistas nas referidas tarefas. Para atribuir uma nota ao aluno em cada atividade, o tutor comparava a resposta apresentada pelo aluno aos critérios de avaliação da atividade. A nota, portanto, representava o quanto o desempenho do aluno, na percepção do tutor, havia atingindo os critérios de avaliação propostos para a atividade.

\section{Coleta e análise dos dados}

Tanto as informações dos alunos como as informações dos tutores foram extraídas do AVA após o término do curso. Os feedbacks dos tutores foram categorizados quanto a: (a) sua presença ou ausência, (b) amplitude, (c) efeito, (d) função e (e) organização. Os dados foram organizados em uma planilha do Excel e análises estatísticas foram realizadas utilizando o Statistical Package for the Social Science (SPSS) versão 20. Devido ao tamanho da amostra, foram utilizados testes estatísticos não-paramétricos (Dancey \& Reidy, 2006).

\section{RESULTADOS E DISCUSSÃO}

Dos 37 cursistas, 14 alunos foram excluídos da presente análise porque não realizaram nenhuma das atividades (6 alunos) ou não concluíram o módulo (8 alunos). Portanto, os resultados que se seguem referem aos dados dos 23 cursistas que concluíram o módulo.

\section{Presença/Ausência de feedback}

Ao longo do módulo foram propostas 20 tarefas. A Tabela 1 apresenta um panorama geral da participação dos cursistas nas tarefas.

Como é possível observar na Tabela 1, destacam-se os seguintes resultados: (a) $65.0 \%$ das tarefas eram do tipo TAC; (b) $80.0 \%$ das tarefas foram respondidas por todos os cursistas que concluíram o módulo (a exceção foram as tarefas T3.3, T4a1,T4a3 e T5.1); (c) o número de cursistas que receberam feedback em cada tarefa variou entre cinco (na T2.1) e 22 (na T6.2); (d) em 75.0\% das tarefas a mediana das notas sem feedback foi maior do que a mediana das notas com feedback (a exceção foram as tarefas T2.1, T2.3, T4b1, T4b2 e T6.2); (e) a diferença foi estatisticamente significativa $(p<0,5)$ apenas nas tarefas T1.1 e T5.2, ambas TAC. Portanto, os resultados parecem sugerir que, de uma maneira geral, não existem diferenças estatisticamente significativas nas notas em função da presença do feedback. No entanto, nos casos em que a diferença foi significativa, os participantes que não tinham feedback receberam notas maiores do que aqueles com feedback.

Também se examinou se havia diferenças na média final do módulo em função da quantidade de tarefas com feedback. Os cursistas foram divididos em três grupos: (a) aqueles que receberam feedback em até três tarefas, (b) aqueles que receberam feedback de 4 a 11 tarefas e (c) aqueles que receberam feedback em 12 tarefas ou mais. O teste de Kruskal Wallis demonstrou que não houve diferenças estatisticamente significativas entre os grupos $\left(x^{2}(2)=1.41, P=0.49\right)$.

A presença/ausência do feedback dos tutores às respostas fornecidas pelos cursistas parece não interferir na nota da maioria das tarefas. Contudo, as tarefas sem feedback, no geral, apresentam notas maiores do que as tarefas com feedback. Um aspecto que parece ser importante destacar é o fato de que o aluno poderia, dentro do prazo de cada unidade, refazer e/ou aperfeiçoar suas respostas, a partir do feedback fornecido pelo tutor, para obter uma nota melhor. Infelizmente, não foi mantido no AVA um histórico dos feedbacks fornecidos pelos tutores para cada uma das tarefas e é possível que o tutor tenha apagado o feedback após o cursista melhorar sua resposta.

Essa hipótese parece ser corroborada por alguns feedbacks que sinalizam que o aluno refez a tarefa e que o tutor está fornecendo um novo feedback, como pode ser observado nos seguintes exemplos: T04: Parabéns pela tarefa refeita!!; T10: Seu exemplo ficou mais completo quando você apresentou mais argumentos para expor sua opinião.; e T12: Parabéns pela tarefa. Você a refez com muito cuidado e usou adequadamente as 
Tabela 1. Panorama geral da participação dos cursistas nas tarefas.

\begin{tabular}{|c|c|c|c|c|c|c|c|}
\hline \multirow{3}{*}{ Unidade } & \multirow{3}{*}{ Tarefa } & \multirow{3}{*}{$\begin{array}{l}\text { Tipo de } \\
\text { Atividade }\end{array}$} & \multirow{3}{*}{$\mathbf{N}$} & \multirow{3}{*}{$\begin{array}{l}\text { Respostas com } \\
\text { feedback }\end{array}$} & \multicolumn{3}{|c|}{ Notas } \\
\hline & & & & & \multicolumn{2}{|c|}{ Mediana } & \multirow{2}{*}{$\begin{array}{l}\text { Teste U de Mann- } \\
\text { Whitney (p) }\end{array}$} \\
\hline & & & & & Com Feedback & Sem Feedback & \\
\hline \multirow{3}{*}{1} & $\mathrm{~T} 1.1$ & TAC & 23 & 11 & 9.05 & 14.71 & $33.50(0.04)$ \\
\hline & $\mathrm{T} 1.2$ & TAC & 23 & 7 & 10.36 & 12.72 & $44.50(0.41)$ \\
\hline & $\mathrm{T} 1.3$ & TIC & 23 & 10 & 11.10 & 12.69 & $56.00(0.58)$ \\
\hline \multirow{3}{*}{2} & $\mathrm{~T} 2.1$ & TIC & 23 & 5 & 12.20 & 11.94 & $44.00(0.94)$ \\
\hline & $\mathrm{T} 2.2$ & TAC & 23 & 7 & 9.36 & 13.16 & $37.50(0.21)$ \\
\hline & $\mathrm{T} 2.3$ & TAC & 23 & 7 & 13.07 & 11.53 & $48.50(0.58)$ \\
\hline \multirow{3}{*}{3} & T3.1 & TAC & 23 & 7 & 10.43 & 12.69 & $45.00(0.42)$ \\
\hline & T3.2 & $\mathrm{TIC}$ & 23 & 7 & 11.86 & 12.06 & $55.00(0.94)$ \\
\hline & T3.3 & TIC & 22 & 8 & 8.56 & 13.18 & $32.50(0.06)$ \\
\hline \multirow{5}{*}{4} & T4a1 & TAC & 22 & 8 & 9.88 & 12.43 & $43.00(0.36)$ \\
\hline & T4a2 & TAC & 23 & 9 & 10.11 & 13.21 & $46.00(0.26)$ \\
\hline & T4a3 & TIC & 22 & 9 & 8.61 & 13.50 & $32.50(0.07)$ \\
\hline & T4b1 & TAC & 23 & 11 & 12.23 & 11.79 & $63.50(0.88)$ \\
\hline & $\mathrm{T} 4 \mathrm{~b} 2$ & TIC & 23 & 10 & 12.35 & 11.73 & $61.50(0.82)$ \\
\hline \multirow{4}{*}{5} & T5.1 & TAC & 21 & 12 & 10.13 & 12.17 & $43.50(0.44)$ \\
\hline & $\mathrm{T} 5.2$ & TAC & 23 & 11 & 7.73 & 15.92 & $19.00(0.01)$ \\
\hline & T5.3 & TAC & 23 & 11 & 9.91 & 13.92 & $43.00(0.14)$ \\
\hline & T5.4 & TIC & 23 & 10 & 10.50 & 13.15 & $50.00(0.33)$ \\
\hline \multirow{2}{*}{6} & T6.1 & TAC & 23 & 10 & 9.25 & 14.12 & $37.50(0.58)$ \\
\hline & T6.2 & TAC & 23 & 22 & 12.32 & 5.00 & $4.00(0.26)$ \\
\hline
\end{tabular}

Nota: TAC - Tarefa Acadêmica de Casa; TIC - Tarefa Interpessoal de Casa; N - Número de cursistas que realizaram a tarefa.

denominações das classes e subclasses de habilidades sociais apresentadas nesta Unidade!!!! Isso poderia justificar, em parte, o fato de: (a) as notas das tarefas sem feedback serem maiores do que as notas das tarefas com feedbacks e (b) não haver diferença estatisticamente significante entre os alunos que possuem no AVA quantidades diferentes de tarefas com feedback. Além disso, a possibilidade de que alguns tutores tenham apagado o feedback após corrigir uma tarefa refeita, pode afetar os demais resultados que serão apresentados a seguir.

\section{Classificação do feedback}

Multiplicando o total de cursistas $(n=23)$ pelo total de tarefas do módulo $(n=20)$, verifica-se que poderiam ser encontrados até 460 feedbacks. Contudo, no momento em que os dados foram extraídos do AVA, havia na plataforma 192 feedbacks, o que representava $41.7 \%$ do total de feedbacks possíveis. O número de feedback por tarefa e por unidade variou. A média de feedback por unidade foi de 32.0 e por tarefa foi de 9.6. As unidades 4 e 5 apresentaram o maior número de feedback, o que era esperado por serem unidades com uma quantidade maior de tarefas, respectivamente, 5 e 4 tarefas.

Os feedbacks foram classificados quanto à amplitude, efeito, função e organização. A Tabela 2 apresenta a distribuição da frequência dos feedbacks em cada uma das classes e subclasses avaliadas. Quanto à amplitude, $79.2 \%$ dos feedbacks foram mais específicos, destacando algum aspecto do desempenho do cursista na tarefa (por exemplo, T06: Você realizou uma boa análise dos vídeos, indicando as justificativas para o desempenho empático e pró-empático e deixando claro que compreendeu o conceito.) ou no curso (por exemplo, T13: Parabéns pela tarefa. Consigo perceber que você se dedica e está aprendendo o que é proposto, porém, é preciso descrever com mais detalhes o contexto e os comportamentos.). Para Archer et al. (2016, p. 478), "ao avaliar o desempenho do estudante, o professor necessita fornecer feedback, o mais preciso e inequivocamente possível, a respeito de quais aspectos de seu desempenho necessitam ser aprimorados, ou estão apropriados". Além disso, de acordo com Z. A. P. Del Prette e Del Prette 
Tabela 2. Distribuição da frequência dos feedbacks em cada uma das classes e subclasses avaliadas.

\begin{tabular}{|c|c|c|c|c|c|c|c|c|c|}
\hline \multirow{2}{*}{ Classificação } & \multirow{2}{*}{ Subcategorias } & \multicolumn{6}{|c|}{ Unidade } & \multirow{2}{*}{ Total } & \multirow{2}{*}{$\%$} \\
\hline & & 1 & 2 & 3 & 4 & 5 & 6 & & \\
\hline \multirow{2}{*}{ Amplitude } & Geral & 6 & 3 & 3 & 9 & 11 & 8 & 40 & 20.83 \\
\hline & Específico & 22 & 16 & 19 & 38 & 33 & 24 & 152 & 79.17 \\
\hline \multirow{5}{*}{ Efeito } & Positivo & 13 & 11 & 13 & 20 & 16 & 16 & 89 & 46.35 \\
\hline & Negativo & 4 & 2 & 2 & 7 & 5 & 1 & 21 & 10.94 \\
\hline & Sanduíche & 4 & 2 & 4 & 9 & 6 & 0 & 25 & 13.02 \\
\hline & Positivo/Negativo & 5 & 3 & 1 & 9 & 16 & 14 & 48 & 25.00 \\
\hline & Negativo /Positivo & 2 & 1 & 2 & 2 & 1 & 1 & 9 & 4.69 \\
\hline \multirow{2}{*}{ Função } & Verificação & 10 & 8 & 9 & 26 & 16 & 20 & 89 & 46.35 \\
\hline & Elaboração & 18 & 11 & 13 & 21 & 28 & 12 & 103 & 53.65 \\
\hline \multirow{2}{*}{ Organização } & Diretivo & 7 & 4 & 5 & 19 & 18 & 13 & 66 & 64.08 \\
\hline & Facilitador & 8 & 4 & 4 & 8 & 10 & 3 & 37 & 35.92 \\
\hline
\end{tabular}

(2010), em um THS, o feedback deve permitir que a pessoa perceba como se comporta e as consequências de sua ação. Assim sendo, uma frequência maior de feedbacks específicos não apenas é esperada, mas também é desejável para gerar resultados melhores e mais precisos no processo de desenvolver e/ou aperfeiçoar as habilidades sociais.

Em relação ao efeito, foram identificadas cinco subclasses de feedback de acordo com a presença de afirmações que valorizavam (positivos) e/ou reprovavam (negativos) aspectos da resposta apresentada pelo cursista. Dos 192 feedbacks presentes no AVA, 46.3\% eram apenas positivos e $15.6 \%$ eram apenas negativos ou iniciavam com aspecto negativo do desempenho do cursista. Z. A. P. Del Prette e Del Prette (2010, p. 69) defendem que o feedback positivo deve ser preferido ao invés do feedback negativo, pois o feedback positivo:

a)evita ressentimentos e reações defensivas, comumente associadas ao feedback negativo;

b)dispõe a pessoa a ouvir com mais atenção as observações feitas pelo interlocutor, ampliando seu conhecimento sobre o próprio desempenho e/ou os resultados dele decorrentes;

c) motiva a pessoa a investir no aperfeiçoamento dos aspectos valorizados;

d)aumenta a probabilidade dos desempenhos valorizados voltarem a ocorrer.

Nessa direção, o feedback sanduíche parece ser o tipo de feedback mais indicado quando é necessário pontuar um aspecto do desempenho do cursista que precisa ser corrigido. Ao usar esse tipo de feedback, o tutor contribui para que o aluno se sinta encorajado, pois a devolutiva fornecida inicia e finaliza com aspectos adequados da resposta e/ou atitudes a serem mantidos (Silva, 2017). Do total de feedbacks presentes no AVA, apenas $13.0 \%$ podiam ser classificados como feedback sanduíche. No entanto, como é possível que muitos feedbacks tenham sido apagados e/ou modificados após o participante refazer sua resposta, essa porcentagem pode não representar a real frequência desse tipo de feedback ao longo do curso.

Quanto à função, 53.6\% dos feedbacks buscavam apresentar dicas e orientações sobre como chegar à resposta esperada, ao invés de apresentar um simples julgamento a respeito de a resposta estar correta ou incorreta. De acordo com Shute (2008), enquanto o feedback de verificação resume-se a informar ao aluno se a resposta está correta ou não, o feedback de elaboração pode caminhar por diversas direções, tais como: (a) direcionar para um tópico ou aspecto do conteúdo abordado pela tarefa, (b) direcionar a resposta para um resultado esperado, (c) discutir erros particulares, (d) apresentar exemplos que contribuam para o aluno compreender melhor o conteúdo e/ou onde encontra-se o equívoco de sua resposta e (e) dar orientação de maneira gentil. Assim sendo, feedbacks de elaboração poderiam contribuir para ampliar a compreensão do conteúdo, bem como para auxiliar o aluno a refletir sobre suas habilidades sociais.

A última categoria de análise refere-se à organização dos feedbacks. Só foram classificados quanto à organização os feedbacks negativos, ou seja, aqueles que reprovavam algum aspecto da resposta do cursista. Essa decisão foi tomada porque essa última forma de classificar os feedbacks está relacionada a como o tutor solicita ao cursista que modifique suas respostas incorretas. Dos 103 feedbacks que envolviam a reprovação de algum aspecto da resposta, $64.1 \%$ apontavam diretamente o que precisava ser corrigido ou revisado. Apenas 35.9\% deles apresentavam comentários e/ou sugestões para orientar o aluno no processo de revisão da tarefa. Para 
Shute (2008), o feedback deveria assumir uma função formativa, ou seja, deveria contribuir para que o aluno melhore seus conhecimentos, habilidades e compreensão em alguma área de conteúdo ou de habilidades. Nesse sentido, os feedbacks diretivos, por especificarem o que precisa ser alterado, são importantes para que os alunos identifiquem de maneira precisa o que em sua resposta diverge do resultado esperado. Já o feedback facilitador parece estar mais relacionado ao processo de conduzir o aluno a uma compreensão mais ampla do que está sendo abordado. Assim, o predomínio de um tipo ou outro de feedback pode depender da percepção do tutor sobre a "gravidade" do erro cometido pelo aluno, da importância atribuída ao tema abordado pela tarefa e da compreensão sobre como o feedback fornecido interfere no processo de desenvolvimento das habilidades sociais. As diferenças encontradas acima podem estar relacionadas a diversidade de tutores que atuaram no módulo.

Também se examinou se havia diferença nas notas, em cada uma das tarefas, devido a alguma dessas características do feedback. Para a amplitude, função e organização empregou-se o Teste $U$ de Mann-Whitney e para o efeito utilizou-se Teste de Kruskal Wallis. Não foram encontradas diferenças estatisticamente significativas em nenhuma das tarefas, com exceção de dois casos. Em T6.2, os cursistas que receberam feedbacks com amplitude geral tiveram notas mais altas (mediana $=18.5$ ) do que cursistas que receberam feedbacks com amplitude específica (mediana $=8.88$ ), $U-6.0$ e $p=$ 0.01 . Já na T6.1, a diferença foi em relação ao efeito do feedback $\left(x^{2}=7.6\right.$ e $\left.p=0.02\right)$. Os cursistas com feedbacks positivos tiveram notas maiores (mediana $=$ 8.00), quando comparados com aqueles que receberam feedbacks positivo/negativo (mediana $=3.2$ ) e aqueles que receberam feedbacks negativo/positivo (mediana $=2.0$ ). Contudo, o tamanho do efeito foi insignificante. Além disso, como não houve acesso ao histórico dos feedbacks fornecido à uma mesma tarefa, esses dados precisam ser tomados com cautela. Novos estudos são necessários para verificar se tais resultados se mantêm.

\section{Tipo de tarefa}

Das 20 tarefas propostas no módulo, 13 eram TACs e sete eram TICs. As TICs tiveram notas mais altas (mediana $=13.1$ ) do que as TACs (mediana $=9.1$ ), mas essa diferença não foi estatisticamente significativa $(U=27.0, p=0.14)$. Pelo menos duas hipóteses podem ser levantadas para tentar explicar esse resultado. Primeiro, é possível que os alunos tenham sentido mais facilidade em realizar as TICs, por serem atividades práticas, do que para realizar as TACs, visto que essas requeriam o domínio de uma área de conhecimento nova para os participantes. Nesse sentido, é possível que os alunos precisassem de um tempo maior para compreender os conceitos e se tornarem aptos para usá-los de maneira coerente.

Uma segunda hipótese refere-se à exigência dos tutores frente ao conteúdo das respostas em cada tipo de tarefa. As TICs envolviam o compartilhamento das experiências pessoais dos participantes, o que pode ter despertado a empatia do tutor em relação às situações apresentadas e afetado as notas atribuídas. Já as TACs requeriam que os alunos demonstrassem sua compreensão a respeito dos conceitos da área e sua capacidade de aplicá-los na análise de situações de interação interpessoal, o que pode ter levado os tutores a adotarem critérios mais exigentes para atribuir notas às respostas fornecidas pelos participantes a essas tarefas.

Em relação à classificação do feedback, só houve diferença estatisticamente significativa entre TICs e TACs em relação ao efeito do feedback. As TACs receberam mais feedbacks negativos (mediana $=12.3$ ) do que as TICs (mediana $=7.2$ ), $U=22.5$ e $p=0.05$. Já as TICS receberam mais feedbacks sanduíche (mediana $=14.2$ ) do que as TACs (mediana $=8.5$ ),$U=19.5$ e $p=$ 0.03 . Esses dois dados parecem corroborar a segunda hipótese apresentada acima.

\section{CONSIDERAÇÕES FINAIS}

O presente estudo configura-se como uma tentativa inicial de avaliar como o feedback dos tutores em THS ofertado na modalidade híbrida pode contribuir para o desenvolvimento das habilidades sociais dos participantes. Uma vez que o processo de aquisição, fortalecimento e manutenção das habilidades sociais possui um caráter eminentemente prático (A. Del Prette \& Del Prette, 2010; Z. A. P. Del Prette \& Del Prette, 2010), uma das principais preocupações consiste em criar condições para que, a partir das atividades propostas no AVA, os alunos possam efetivamente desenvolver tais habilidades. Nesse sentido, o feedback fornecido pelo facilitador desempenha um papel importante no processo de aprendizagem.

A principal contribuição do estudo foi apresentar uma proposta inicial para analisar os feedbacks dos tutores disponíveis no AVA. Como foi possível observar, o feedback pode ser avaliado de acordo com sua presença ou ausência, amplitude, efeito, função e organização. Cada uma dessas formas de classificar e avaliar o feedback ajuda a refletir sobre os seus efeitos sobre o desempenho do aluno. Segundo Archer et al. (2016, p. 475), "o uso inapropriado do feedback, ou a não utilização de feedback em avaliações de desempenho implica no aumento da probabilidade de que o egresso de um programa de ensino apresente repertório comportamental limitado que pode vir a trazer consequências para qualidade de sua atuação profissional".

Os resultados obtidos sugerem algumas relações entre os tipos de feedback e o desempenho dos alunos. Contudo, o estudo possui três limitações importantes. A 
primeira é o tamanho da amostra que, por ser pequeno, impede o uso de testes estatísticos mais robustos. A segunda limitação refere-se ao fato de o desempenho dos alunos ter sido avaliado por diferentes tutores, contribuindo para que a comparação entre as notas dos alunos pudesse ser influenciada por um componente subjetivo: a percepção do tutor a respeito do desempenho do aluno. Por fim, a última limitação diz respeito à possibilidade de que alguns feedbacks tenham sido apagados após o aluno refazer a tarefa e melhorar seu desempenho.

Na tentativa de corrigir essas limitações, sugere-se para estudos futuros: (a) aumentar o tamanho da amostra; (b) aumentar o número de alunos por tutor para comparar as notas de diferentes cursistas avaliados por um mesmo tutor; e (c) criar mecanismos para manter um histórico dos feedbacks fornecidos pelos tutores às respostas dos cursistas. Além disso, para tentar eliminar o componente subjetivo das notas atribuídas aos alunos, é possível pensar em criar um sistema de classificação das respostas dos cursistas em função dos objetivos propostos para a tarefa (por exemplo, tarefas que atenderam totalmente, parcialmente ou não atenderam os critérios de avaliação).

Novos estudos também podem avaliar outros aspectos envolvidos no feedback, tais como presença de emoticons, o uso de recursos tipográficos (por exemplo, combinação de maiúsculas e minúsculas, negrito, itálico), o tom produzido na mensagem a partir da análise da escolha das palavras utilizadas, o tempo entre a resposta e o fornecimento de feedback pelo tutor (Abreu-e-Lima \& Alves, 2011). Ademais, a análise de medidas de conhecimento e de repertório de habilidades sociais dos participantes aplicadas antes e após a realização do curso podem contribuir para uma visão mais ampla do efeito feedback sobre o desempenho cognitivo e o desenvolvimento das habilidades sociais dos alunos.

\section{REFERÊNCIAS}

Abed, A. L. Z. (2016). O desenvolvimento das habilidades socioemocionais como caminho para a aprendizagem e o sucesso escolar de alunos da educação básica. Construção Psicopedagógica, 24(25), 8-27.

Abreu-e-Lima, D. M.; Alves, M. N. (2011). O feedback e sua importância no processo de tutoria a distância. ProPosições, 22(2), 189-205.

Archer, A. B.; Crispim, A. C.; Cruz, R. M. (2016). Avaliação e feedback de desempenho de estudantes na Educação a Distância. Avances En Psicología Latinoamericana, 34(3), 473-485. https://doi.org/10.12804/apl34.3.2016.03

Corrêa, C. I. M. (2008). Habilidades sociais e educação: programa de intervenção para professores de uma escola pública (Tese de doutorado). Universidade Estadual Paulista Júlio de Mesquita Filho, Marília, SP.

Dancey, C. P.; Reidy, J. (2006). Estatística sem matemática para psicologia: usando SPSS para Windows (3a ed). Porto Alegre: Artmed.

Del Prette, A.; Del Prette, Z. A. P. (2010). Programa vivencial de habilidades sociais: características sob a perspectiva da análise do comportamento. In Garcia, M. R.; Abreu, P.; Cillo, E. N. P. de; Faleiros, P. B.; Queiroz, P. P. (Eds.), Sobre comportamento e cognição: terapia comportamental e cognitivas (pp. 127-150). Santo André, SP: ESETec.

Del Prette, A.; Del Prette, Z. A. P. (2011). Enfoques e modelos de treinamento de habilidades sociais. In Del Prette A.; Del Prette, Z. A. P. (Eds.), Habilidades sociais: intervenções efetivas em grupos (pp. 19-56). São Paulo: Casa do Psicólogo.

Del Prette, A.; Del Prette, Z. A. P. (2017a). Competência social e habilidades sociais: manual teórico-prático. Petrópolis, RJ: Vozes.

Del Prette, A.; Del Prette, Z. A. P. (2017b). Habilidades sociais de comunicação e feedback. In Del Prette, Z. A. P.; Del Prette, A. (Eds.), Habilidades sociais e competência social para uma vida melhor (pp. 60-68). São Paulo: EdUFSCar.

Del Prette, Z. A. P. (2016). Educação à distância: articulando a promoção de habilidades sociais educativas do professor ao desenvolvimento socioemocional dos alunos na escola. São Carlos: Manuscrito não publicado.

Del Prette, A.; Del Prette, Z. A. P.; Torres, A. C.; Pontes, A. C. (1998). Efeitos de uma intervenção sobre a topografia das habilidades sociais de professores. Psicologia Escolar $e$ Educacional, 2(1), 11-22. https://doi.org/10.1590/S141385571998000100002

Del Prette, Z. A. P.; Del Prette, A. (1997). Um programa de desenvolvimento de habilidades sociais na formação continuada de professores. In CD-Rom dos trabalhos selecionados para apresentação, 20a. Reunião Anual da ANPED (pp. 1-31). Caxambu, MG: ANPED.

Del Prette, Z. A. P.; Del Prette, A. (1999). Psicologia das habilidades sociais: terapia, educação e trabalho. Petrópolis, RJ: Vozes.

Del Prette, Z. A. P.; Del Prette, A. (2009). Avaliação de habilidades sociais: bases conceituais, instrumentos e procedimentos. In Del Prette, Z. A. P.; Del Prette, A. (Eds.), Psicologia das habilidades sociais: diversidade teórica $e$ suas implicações (pp. 189-231). Petrópolis, RJ: Vozes.

Del Prette, Z. A. P.; Del Prette, A. (2010). Psicologia das relações interpessoais: vivências para o trabalho em grupo (8a ed). Petrópolis, RJ: Vozes.

Del Prette, Z. A. P.; Del Prette, A. (2011). Práticas baseadas em evidência e treinamento de habilidades sociais. In Del Prette, A.; Del Prette, Z. A. P. (Eds.), Habilidades sociais: intervenções efetivas em grupos (pp. 57-82). São Paulo: Casa do Psicólogo.

Del Prette, Z. A. P.; Del Prette, A.; Garcia, F. A.; Silva, A. T. B.; Puntel, L. P. (1998). Habilidades sociais do professor em sala de aula: um estudo de caso. Psicologia: Reflexão $e$ Crítica, 11(3), 591-603. https://doi.org/10.1590/S010279721998000300016 
Domitrovich, C. E.; Durlak, J. A.; Staley, K. C.; Weissberg, R. P.(2017). Social-Emotional Competence: An Essential Factor for Promoting Positive Adjustment and Reducing Risk in School Children. Child Development, 88(2), 408-416. https://doi.org/10.1111/cdev.12739

Mill, D. (2017). Polidocência: configurações para a docência na educação a distância. São Carlos: Editora Pixel.

Murta, S. G. (2005). Aplicações do treinamento em habilidades sociais: análise da produção nacional. Psicologia: Reflexão e Crítica, 18(2), 283-291. https://doi.org/10.1590/S010279722005000200017

Rosin-Pinola, A. R.; Marturano, E. M.; Elias, L. C. D. S.; Del Prette, Z. A. P. (2017). Ensinando habilidades sociais educativas para professores no contexto da inclusão escolar. Revista Educação Especial, 30(59), 737-749. https://doi.org/10.5902/1984686X28430

Shute, V. J. (2008). Focus on formative feedback. Review of Educational Research, 78(1), 153-189. https://doi. org/10.3102/0034654307313795

Silva, A. P. R. (2017). Sistema de tutoria e modelos de feedback. São Carlos: Editora Pixel.

Vaughan, N. D.; Cleveland-Innes, M.; Garrison, D. R. (2013). Teaching in blended learning environments: crating and sustaining communities of inquiry. Edmonton, Canadá: AU Press.

Vila, E. M. (2005). Treinamento de habilidades sociais em grupo com professores de crianças com dificuldades de aprendizagem: uma análise sobre procedimentos e efeitos da intervenção (Dissertação de Mestrado). Universidade Federal de São Carlos.

Weissberg, R. P.; Durlak, J. A.; Domitrovich, C. E.; Gullotta, T. P. (2015). Social and emotional learning: Past, present, and future. In Durlak, J. A.; Domitrovich, C. E.; Weissberg, R. P.; Gullotta, T. P. (Eds.), Handbook of social and emotional learning: Research and practice (pp. 3-19). New York, NY: Guilford.

O presente trabalho foi realizado com apoio da Coordenação de Aperfeiçoamento de Pessoas de Nível Superior - Brasil (CAPES) - Código de Financiamento 001.

Recebido: 27 de novembro de 2018

Aprovado: 23 de dezembro de 2019 